\title{
Acknowledgement of Reviewers 2020
}

\section{George Tsakiris ${ }^{1}$}

Published online: 17 February 2021

(C) The Author(s), under exclusive licence to Springer Nature B.V. part of Springer Nature 2021

Please join us in thanking all those scientists and experts in the various fields represented in Water Resources Management for devoting time and effort to review the papers that we have been sending them The Editor-in-Chief, the editorial board and publisher acknowledge the colleagues listed below for their excellent reviews of papers for which final decisions have been made during the period 1 January 2020 to 31 December 2020.

Vasan A

Omid A. Yamini

Jaber Aazami

Aminuddin Ab Ghani

Hassan Abbas

Ali Abbasi

Mohammad Abbasian

Fahmy Abdelhaleem

Brahim Abdelkebir

Dina Abdelmoneim

Fadi Abdelradi

Isam Abdulhameed

Ali Abdullah

G.R. Abhijith

João Abrantes

Adeshina Adebanjo

Warith Adebisi

Blal Adem Esmail

Adeniyi Adeogun

Shahzada Adnan

Okechukwu Agbasi
Mohammad Aghayi
Pouya Aghelpour
V. Agilan
Abdellatif Ahbari
Ishtiyaq Ahmad
Sakinat Ahmad
Mohammad Ahmadi
Iman Ahmadianfar
Ali Ahmed
Ferdous Ahmed
Zhipin Ai
Saeid Akbarifard
Fazlullah Akhtar
Muhanned Al Murib
Ali Al Rammahi
Angelos Alamanos
Yousif Al-Aqeeli
Sadik Alashan
Seyedhassan Alavinia

Okechukwu Agbasi

Mohammad Aghayi

Pouya Aghelpour

Agilan

Abdellatif Ahbari

Ishtiyaq Ahmad

Mohammad Ahmadi

Iman Ahmadianfar

Ahmed

Ferdous Ahmed

Zhipin Ai

Saeid Akbarifard

Muhanned Al Murib

Angelos Alamanos

Yousif Al-Aqeel

Seyedhassan Alavinia

\author{
Mohammad Albaji \\ Tahmineh Aldaghi \\ Ihab Alfadhel \\ Amani Alfarra \\ Andesikuteb Ali \\ Imran Ali \\ Md Ali \\ Zulfiqar Ali \\ Farhad Alizadeh
}

Mohamad Javad Alizadeh

Ahmed Mohammed Sami

Al-Janabi

Jafar Al-Jawad

Loai Aljerf

Ahmed Aljuaidi

Jaber Alkasseh

Mahmoud Al-Khafaji

Sohaib AL-Mamoori

Lucia Almeida

Mustafa Al-Mukhtar

George Tsakiris

gtsakir@central.ntua.gr

1 Lab of Reclamation Works and Water Resources Management, National Technical University, Athens, Greece 


Basim Al-Obaidy
Emran Alotaibi
Samia Aly
Babak Amirataee
Vahab Amiri
Maoui Ammar
Caleb Amos
Jayanath Ananda
Gholamreza Andalib
Panagiotis Angelidis
Mohammad Arab Amiri
Nazia Arbab
Vicky Ariyanti
Ali Arman
Asaad Armanuos
Elisa Arnone
Mohit Arora
Arfan Arshad
Vasan Arunachalam
Mohsen Asadi
Morteza Asadi
Antonia Asencio
Muhammad Ashraf

Seyed Mohammad Ashrafi

Hasnat Aslam

Diego Avesani

Ayman Awadallah

Taymoor Awchi

Alper Aydemir

Francisco Ayuga

Hazi Azamathulla

Hazi Mohammad

Azamathulla

Arash Azari

Asghar Azizian

Elmi Azmi

Monte Azmi

Venkatesh B

K.S. Jinesh Babu

Pius Babuna

Hanne Bach

Lina Bagdziunaite-

Litvinaitiene

Musiime Bahati

Hua Bai

Peng Bai

Tao Bai
Tarate Suryakant Bajirao

Mahendra Baniya

Marcelle Baptista

Reza Barati

Ramon Barberan

Alina Barbulescu

Emanuele Barca

Eyyup Basakin

George Bathrellos

Yıldırım Bayazıt

Subhrasita Behera

Alemshet Belayneh

Rossano Belladona

Vasilis Bellos

Loucif Benahmed

Hassan Benfetta

Mehdi Bensaid

Marco Berardi

Maria Bermudez

Barasa Bernard

Florian Betz

Nirman Bhagat

Birendra Bharti

Sami Bhat

Rajan Bhatt

Chandrashekhar Bhuiyan

Seyed Mostafa Biazar

Doan Binh

Robin Biswas

Bosko Blagojevic

Claudio Blanco

Rewa Bochare

Brunella Bonaccorso

Charles Bong

Charles Bong

Ilke Borowski

Petre Bretcan

Christopher Brown

Bruno Brunone

Elisa Brussolo

Mateus Bueno

Halil Ibrahim Burgan

Naci Büyükkaracığan

C.D. Singh

Jiabing Cai

Jialiang Cai

Xiatong Cai
Jose Ruben Campos-Gaytan

Laura Canno Ferreira Fais

Shengle Cao

María Carmen Carnero

Silvia Carpitella

Andreza Carvalho

Stefano Casadei

Giulio Castelli

Jesica Castillo-Rodríguez

Srikumar Cattopadhyay

Luana Centeno

Cem Cetinkaya

Praveenkumar Ch

Cristian Chadwick

Hongxiang Chai

Jacob Chandapillai

Jianxia Chang

Xuexiang Chang

Winai Chaowiwat

Barkha Chaplot

Nikos Charizopoulos

K.W. Chau

Kwok-Wing Chau

Adriadna Del Socorro

Chávez Jiménez

Da-Chun Chen

Jui-Sheng Chen

Pan Chen

Xiaojiang Chen

Ying-Jung Chen

Yuanfang Chen

Shin-Jen Cheng

Kong Chhuon

Po-Kuan Chiang

Pascale Chiron

Suryanarayana Chodavarapu Jung Hyun Choi

Bahram Choubin

AFM Kamal Chowdhury

Shahadat Chowdhury

Eliane Da Christo

Il-Moon Chung

Gustavo Coelho

Marcelo Coelho

Theodora Cohen Liechti

Virginia Rosa Coletta

Peter Cookey 


\begin{tabular}{|c|c|c|}
\hline Juan Ignacio Corcoles & Ahmed Elbeltagi & Bruna Gerônimo \\
\hline Romulus-Dumitru Costache & Alper Elci & Brook Getahun \\
\hline Enrico Creaco & Hisham Eldardiry & Amir Ghaderi \\
\hline Huijuan Cui & Mutasem El-Fadel & Mona Ghafouri-Azar \\
\hline Thibaut Cuvelier & Mohamed El-Mahdy & Roghayeh Ghasempour \\
\hline Mônica Da Hora & Ali El-Mahfoodh & Reza Ghazavi \\
\hline Alexandre Da Silva & Ahmed El-Shafie & Vahid Gholami \\
\hline Chao Dai & Mohamed Eltarabily & Mohammad Ali Ghorbani \\
\hline Huseyin Dalkilic & Alireza Emadi & Dillip Ghose \\
\hline Sathish Kumar Damodaran & Mohammad Emami & Nesrine Ghouili \\
\hline Srikanth Satish Kumar Darapu & Somayeh Emami & Dickson Gidion \\
\hline Alireza Dariane & Tarkan Erdik & Margarita Gil Samaniego \\
\hline Moustafa Darweesh & Marina Erechtchoukova & Ramos \\
\hline Jew Das & Venkata Rathnam Erva & Carlo Giudicianni \\
\hline Sayan Das & Morteza Esfandyari & Erfan Goharian \\
\hline Fernando Das Gracas Braga & Masoud Eshghizadeh & Mohammad Hossein \\
\hline Da Silva & Rasha Fadhil & Golmohammadi \\
\hline Josef Datel & M.D. Golam Rabbani Fahad & Ricardo Gomes \\
\hline J. L. M. P. De Lima & Muhammad Abrar Faiz & Yicheng Gong \\
\hline Luca De Oliveira Turci & Fernando Fan & Ana Cristina González \\
\hline Thushara De Silva M & Yubing Fan & Valoys \\
\hline Surajit Deb Barma & Gonghuan Fang & Francisco González-Gómez \\
\hline Majid Dehghani & Shiqi Fang & Rebeca González-Villela \\
\hline Benjamin Wullobayi & Elias Farah & Mustafa Goodarzi \\
\hline Dekongmen & Alireza Farid Hoseini & Ashantha Goonetilleke \\
\hline Roberto Del Teso March & Nima Fayaz & Ajanta Goswami \\
\hline Hugo Delottier & João Fernandes & Chandre Gowda C \\
\hline Nicholas Dercas & Irene Fernández García & Manish Kumar Goyal \\
\hline Gordana Devic & Marco Ferrante & Alfredo Granados \\
\hline Suhas Dhande & Wieslaw Fialkiewicz & Michele Greco \\
\hline Arun Dhawale & Misbah Fida & Ali Gul \\
\hline Danyang Di & Lachezar Filchev & Mert Gunacti \\
\hline Slava Dineva & Elham Firouznia & Sushindra Gupta \\
\hline Victor Emanuel Diniz & Adriana Flores & Vivek Gupta \\
\hline António Diogo & Tedlaye Forsido & Carlos Gutiérrez-Martín \\
\hline Funda Dökmen & Micael Fraga & M. Habib-Ur-Rehman \\
\hline Mehri Dolatshahi & Gabriele Freni & Sezgin HacısalihoğLu \\
\hline Souag-Gamane Doudja & Yicheng Fu & Zahreddine Hafsi \\
\hline T.K. Drissia & Thais Fujita & Fereshte Haghighi Fashi \\
\hline H.F. Duan & Mohamad Ali Fulazzaky & Didier Haguma \\
\hline Weili Duan & Ivo Fustos & Mohammed Hajeeh \\
\hline Anabela Durão & Mohamed Gabr & Wiktor Halecki \\
\hline Rebecca Dziedzic & Imen Gam & Ivan Halkijevic \\
\hline Naser Ebadati & Dariusz Gąsiorowski & Julia Hall \\
\hline Elham Ebrahimi & Savia Gavazza & Hanamant Halli \\
\hline Davidson Egirani & Jany George & Faiza Hallouz \\
\hline Firat Ekinci & Pantazis Georgiou & Moshrik Hamdi \\
\hline
\end{tabular}


Mohammad Hamed

Aadil Hamid

Yongming Han

David Haro Monteagudo

Waqed Hassan

Mohammad Reza

Hassanvand

István Hatvani

Shakeel Hayat

Binghui $\mathrm{He}$

Ran-Ran He

Shaokun $\mathrm{He}$

Xin $\mathrm{He}$

Yanhu $\mathrm{He}$

Salim Heddam

Hans Jørgen Henriksen

Younggu Her

Mahdi Hesami Afshar

Ganesh Hinge

Farnaz Hooshmand

Marjan Hosseini

Farzad Hosseini Hossein

Abadi

Seyed-Mohammad

Hosseini-Moghari

Zeinab

Hosseinzadehghazichaki

Eva Audrey Yessito

Houessou-Dossou

V. Hrissanthou

Zhigen $\mathrm{Hu}$

Xiaorong Huang

Yenchen Huang

Vijiraja Ilayarajan

Ali Reza Ildoromi

Aleksandra Ilić

Indarto Indarto

Shashi Indwar

Ahmet Irvem

Adlul Islam

Md Monirul Islam

Muktarun Islam

Ardalan Izadi

Azizallah Izady

Jafar Jafrai-Asl

Chowdhury Jahan

Hamidreza Jahangirzadeh
Babak Jahani

Geoffery James Gerusu

Rehan Jamil

Farmanullah Jan

Jiun-Huei Jang

Piyapong Janmaimool

Tyler J Jarvis

Tehseen Javed

Ponselvi Jeevaragagam

Hatem Jemmali

Bing-Chen Jhong

Benyou Jia

Zhonghua Jia

Zhiqiang Jiang

Naveen Joseph

V. Jothiprakash

Konstantinos Kaffas

Taher Kahil

Pradip Kalbar

Koosha Kalhor

Mohammad Ismaeil Kamali

Mahirah Kamaludin

Reza Kamgar

Kandianawati Kandianawati

Shamsa Kanwal

Thokozani Kanyerere

Omid Karami

Ertugrul Karas

Masoud Karbasi

Hamid Kardan Moghaddam

Neamat Karimi

Keivan Karimlou

Zahra Kashkaki

Samir Kateb

Manpreet Kaur

Elcin Kentel

Mulu Kerebih

Hamed Ketabchi

Mohammad Reaza Khaleghi

Saeid Khalifeh

Bahaa Khalil

Mohd Yawar Khan

Zohreh Khorsandi

Kouhanestani

Anthony Kiem

Daeha Kim

Hyun Woo Kim
Sang Ug Kim

Sungwon Kim

Yong Gun Kim

Umut Kırdemir

Krzystof Kochanek

Nagesh Kolagani

Venkatesh Kolluru

Georgios Koulinas

Mohammad Reza Kousari

Georgios N Kouziokas

Cihangir Koycegiz

Akhouri Pramod Krishna

Katarzyna Kubiak-Wójcicka

Justyna Kubicz

Nanabhau Kudnar

Ashok Kumar

Dilip Kumar

K. Satish Kumar

Navsal Kumar

Neeraj Kumar

Pawan Kumar

Prashant Kumar

Ramesh Kumar

Vijendra Kumar

Alban Kuriqi

Bharat Kusre

Lukasz Kuzminski

Claudia Lardizabal

Sarmad Dashti Latif

D. Latinopoulos

Dionissis Latinopoulos

William Laton

Congying Li

Fawen Li

Hong Li

Hui Li

Jian Li

Kailong Li

Rongbo Li

Tao Li

Xiangquan Li

Xungui Li

Ying Li

Xiao Liang

Hebin Lin

Kairong Lin

Hayat Lionboui 


\begin{tabular}{|c|c|c|}
\hline Bojun Liu & Farid Miarnaeimi & Jacob Noble \\
\hline Fei Liu & Ivan Milojković & Mahsa Noori \\
\hline Guihua Liu & Milad Mirzaei Aminiyan & M.Masud Noruzi \\
\hline Jiajia Liu & Numan Mizyed & Eric Noubissié \\
\hline Shuming Liu & Dariusz Młyński & Tiago Novais \\
\hline Yang Liu & Sanaz Moghim & Chrysoula Ntislidou \\
\hline Yong Liu & Mahmoud Mohammad & Martine Nyeko \\
\hline Yuyu Liu & Rezapour Tabari & Muritala Oke \\
\hline Adam Loch & Sankar Mohan & Padam Omar \\
\hline Athanasios Loukas & Ghazaleh Mohebbi Tafreshi & Mohammed Omer \\
\hline Huiting Lu & Jose-Luis Molina & Fernando Oñate-Valdivieso \\
\hline Bin Luo & Biswajit Mondal & Ibrahim Oroud \\
\hline Mingjie Luo & Prolay Mondal & Kaveh Ostad-Ali-Askari \\
\hline Pingping Luo & Majid Montaseri & Avi Ostfeld \\
\hline Yun Luo & Pilar Montesinos & Hesbon Otieno \\
\hline Zengliang Luo & Amir Mohammad Moradi & Ayfer Özdemir \\
\hline Janga Reddy M. & Mojtaba Moravej & Sreeja P. \\
\hline Qiang MA & Hugo Augusto Marinho & Sudheer Padikkal \\
\hline U. Maalik & Moreira & Rebecca Page \\
\hline Aline Machado & Wallisson Moreira De & Dinagara Pandi \\
\hline Deepesh Machiwal & Carvalho & Georgia Papacharalampous \\
\hline Mohamad Reza Madadi & Mohamed Mostafa & Dimitris Papadimos \\
\hline Gohar Mahar & Sayed Masood Mostafavi & Daeryong Park \\
\hline Wael Mahmod & Darani & Atefeh Parvaresh Rizi \\
\hline Adelena Maia & Elaheh Motevalibashi & Dhruvesh Patel \\
\hline Bahram Malekmohammadi & Naeini & Shivaji Patil \\
\hline Shaikh Abdullah Al Mamun & S. Hooman Mousavi & Ahmad Payab \\
\hline Hossain & Ahmed Moussa & David Peres \\
\hline Despoina Manouseli & Srinivas Mudrakartha & Miguel Ángel Pérez-Martín \\
\hline Jeffrey Denzil Marak & Habib Muhammetoglu & Modesto Pérez-Sánchez \\
\hline Athanasios Maramathas & Christos Myriounis & George P. Petropoulos \\
\hline Martina Mario & Ramsundram N & Andrea Petroselli \\
\hline Milica Markovic & Xiaodong $\mathrm{Na}$ & Dai Pham \\
\hline Annlize Marnewick & Ali Reza Nafarzadegan & Sabreen Pibars \\
\hline Araceli Martin-Candilejo & Mohammad Najafzadeh & Katarzyna Pietrucha- \\
\hline Faizan Masoodi & I. Nalbantis & Urbanik \\
\hline Siti Mas'udah & Boini Narsimlu & Ahmadreza Pirali Zefrehei \\
\hline Herlander Mata-Lima & Ali Nasser & Jamshid Piri \\
\hline Luis Mateo & Rozina Naz & Erik Porse \\
\hline Gagan Matta & Mehdi Nezhad Naderi & Maria Manuela Portela \\
\hline Jonatas Mattos & Romeji Ngangbam & Maria Manuela Portela \\
\hline Alexandros Maziotis & Cosmo Ngongondo & Yavar Pourmohamad \\
\hline Joana Medeiros & Xiaokuan Ni & M.N. Prakash \\
\hline Luis Mediero & Majid Niazkar & A.D. Prasad \\
\hline Darshan Mehta & Tewodros Assefa Nigussie & Pooja Preetha \\
\hline Nazli Mehzad & Alireza Nikbakht Shahbazi & Virendra Proag \\
\hline Shein Mezour & Olga Nitcheva & Mario Procopiuck \\
\hline
\end{tabular}




\begin{tabular}{|c|c|c|}
\hline Aris Psilovikos & Edivaldo Serrão & Guilherme Teixeira \\
\hline Valeria Puleo & Pelin Sertyeșilișık & Meseret Teweldebrihan \\
\hline Farhad Qaderi & Sadra Shadkani & Dimitris Tigkas \\
\hline Huanhuan Qin & Hosna Shafaei & Nity Tirkey \\
\hline Sujay Raghavendra N & Kazem Shahverdi & Slavisa Trajkovic \\
\hline Md Salman Rahman & Mohammad Reza Sharifi & Matteo Trigatti \\
\hline Majeed Ramal & Uttam Sharma & Mahesh Tripathi \\
\hline Venkata Ramana Rambha & Xiaojing Shen & Flavio Trojan \\
\hline Yousef Ramezani & Farooq Sher & Demetrios Tsesmelis \\
\hline Sandra Ramos & Haiyun Shi & Qin Tu \\
\hline Rezan Rasheed & Haoran Shi & Mustafa Tunc \\
\hline Abhikarsha Ray & David Ching-Fang Shih & Yunfei Tuo \\
\hline Suresh Reddy & Ehsan Shirangi & Veysel Turan \\
\hline Dattatray Regulwar & Mojtaba Shourian & Evren Turhan \\
\hline Imane Rhouch & Narayan Shrestha & Duygu Ulker \\
\hline Hossien Riahi Madvar & Pedro Silva & Koichi Unami \\
\hline Jose Ribas & Ajay Singh & Babak Vaheddoost \\
\hline Carolina Ribeiro & Ajit Pratap Singh & Mahdi Valikhan Anarak \\
\hline Syed Shams Ahmad Rizvi & Deepak Singh & Divyesh Varade \\
\hline Jéssica Rodrigues & Gagandeep Singh & Deepa Varghese \\
\hline Roberto Roson & Leelambar Singh & Vahid Varshavian \\
\hline Giuseppe Rossi & Prafull Singh & Rambha Venkataramana \\
\hline Mousumi Roy & Raj Singh & Lorenzo Vergni \\
\hline Fouad Saeed & Uttam Singh & Giacomo Viccione \\
\hline Hamid Safavi & Nuanchan Singkran & Matej Vojtek \\
\hline A.Ufuk Sahin & Behrouz Sobhani & Alejandra Volpedo \\
\hline Madhumita Sahoo & Adebayo Sojobi & Isaac Wait \\
\hline Amir Hossein Salimi & Jaysukh Songara & Andrzej Wałęga \\
\hline Abrari Salleh & Paula Soto Rios & Thomas Walski \\
\hline Mahsa Samadi-Darafshani & Konstantinos Soulis & Cheng Wang \\
\hline Saad Shauket Sammen & Mike Spiliotis & Fei Wang \\
\hline Francisco-Javier Sánchez- & Shanmukha Srinivas G & Hongrui Wang \\
\hline Romero & Sunil Srivastava & Jingcai Wang \\
\hline David Santillan Sanchez & Fuzieah Subari & Jinwen Wang \\
\hline Juliano Santos Finck & Jueyi Sui & Lei Wang \\
\hline Luis Sañudo-Fontaneda & Sadeq Oleiwi Sulaiman & Lili Wang \\
\hline Marios Sapountzis & Ali H Ahmed Suliman & Shun-Jiu Wang \\
\hline Anindita Sarkar & Qamar Sultana & Wenbing Wang \\
\hline Sandip Sathe & Fengyun Sun & Yuqiu Wang \\
\hline Giovanni M. Sechi & Peng Sun & Melissa Watanabe \\
\hline Marcelo Secron & Zhandong Sun & Huaibin Wei \\
\hline Ahmed Sefelnasr & Ruhhee Tabbussum & Tianfu Wen \\
\hline Décio Semensatto Junior & Wardah Tahir & Ryan Williams \\
\hline Jotham Sempewo & Yaogeng Tan & Yong Jie Wong \\
\hline H.S. Sen & Tiku Tanyimboh & Jianfeng Wu \\
\hline Soumita Sengupta & Reza Tarinejad & Peipeng $\mathrm{Wu}$ \\
\hline Arsalan Sepehri & Hasan Tatli & Susanne Wuijts \\
\hline
\end{tabular}




$\begin{array}{lll}\text { Bo Xiao } & \text { Sarvin Zamanzad Ghavidel } & \text { Wenjing Zhang } \\ \text { Yang Xiao } & \text { Mohanna Zarei } & \text { Bin Zhao } \\ \text { Ankai Xu } & \text { Abhijit Zende } & \text { Binghui Zheng } \\ \text { Mehdi Yaltaghian Khiabani } & \text { Hayati Zengin } & \text { Han Zhou } \\ \text { Walter Yana- De La Riva } & \text { Luxin Zhai } & \text { Jian-Lan Zhou } \\ \text { Zaher Yaseen } & \text { Yuanzheng Zhai } & \text { Liangjun Zhu } \\ \text { Mohammad Reza Yazdani } & \text { Bing Zhang } & \text { Yilin Zhuang } \\ \text { Lei Ye } & \text { Chen Zhang } & \text { Elvis Žic } \\ \text { Burhan Yildiz } & \text { Fei Zhang } & \text { Cornélio Zolin } \\ \text { Baris Yilmaz } & \text { Liang Zhang } & \text { Li Zongjie } \\ \text { Meihua Yuan } & \text { Min Zhang } & \text { Qiang Zou } \\ \text { Mehmet Ali Yurdusev } & \text { Ting Zhang } & \text { Qiting Zuo } \\ \text { Vsevolod Yutsis } & \text { Wei Zhang } & \end{array}$

We apologize for any errors or inadvertent omissions.

Publisher's Note Springer Nature remains neutral with regard to jurisdictional claims in published maps and institutional affiliations. 